\title{
Accurate identification of clinically important Acinetobacter spp.: an update
}

\author{
Saranya Vijayakumar ${ }^{1}$, Indranil Biswas ${ }^{2}$ \& Balaji Veeraraghavan*,1 \\ ${ }^{1}$ Department of Clinical Microbiology, Christian Medical College, Vellore 632 004, Tamil Nadu, India \\ ${ }^{2}$ Department of Microbiology, Molecular Genetics \& Immunology, University of Kansas Medical Center, Kansas City, KS 66160, USA \\ *Author for correspondence: Tel.: +0416 228 2588; Mobile: +91 944221 0555; Fax: +91 416223 2103; vbalaji@cmcvellore.ac.in
}

Acinetobacter species have emerged as one of the most clinically important pathogens. The phenotypic techniques which are currently available are insufficient in accurately identifying and differentiating the closely related and clinically important Acinetobacter species. Here, we discuss the advantages and limitations of the conventional phenotypic methods, automated identification systems, molecular methods and MALDI-TOF in the precise identification and differentiation of Acinetobacter species. More specifically, several species of this genus are increasingly reported to be of high clinical importance. Molecular characterization such as of bla OXA-51 -like PCR together with $r p o B$ sequencing has high discriminatory power over the conventional methods for Acinetobacter species identification, especially within the Acinetobacter calcoaceticus-Acinetobacter baumannii complex.

Lay abstract: Acinetobacter species are considered to be one of the most important pathogens and associated with increased mortality. The species within the Acinetobacter calcoaceticus-Acinetobacter baumannii complex have emerged as high priority pathogens, especially in intensive care units, thereby posing a challenge to infection management practices. However, identification of Acinetobacter to the species level is difficult. Clear differentiation among various Acinetobacter species with available standard biochemical methods and automated systems is challenging. Although various molecular methods are available, they are not regularly used in diagnostic laboratories. The advantages and disadvantages of different methods useful in the accurate identification of Acinetobacter species are discussed in this review.

First draft submitted: 24 December 2018; Accepted for publication: 11 March 2019; Published online: 27 June 2019

Keywords: ACB complex $\bullet$ Acinetobacter baumannii $\bullet$ MALDI-TOF $\bullet$ matrix-assisted laser desorption ionization-time of flight mass spectrometry $\bullet$ molecular methods $\bullet$ phenotypic methods

The genus Acinetobacter includes a heterogeneous group of bacteria that have been considered 'the most troublesome' by clinicians and scientists [1]. This genus has undergone substantial taxonomic modification. Classical phenotypic methods like biochemical tests have proven to be difficult at the species-level identification of Acinetobacter [2].

Currently, the genus Acinetobacter comprises 59 different species with distinct names (www.bacterio.net/acinetobacter.html; last accessed on October 2018). A total of 11 effectively but not validly published named species and nearly 15 tentative species with designation have been reported (http://apps.szu.cz/anemec/anemec.html) [3].

Initially, the Acinetobacter calcoaceticus-Acinetobacter baumannii complex (ACB complex) comprised four species: Acinetobacter calcoaceticus (genomic species 1), Acinetobacter baumannii (genomic species 2), Acinetobacter pittii (previously named as genomic species 3) and Acinetobacter nosocomialis (previously named as genomic species 13 TU). Recently, two new species, Acinetobacter seifertii (previously known as genomic species close to $13 \mathrm{TU}$ ) and Acinetobacter dijkshoorniae (closely related to $A$. pittii) were also included within the ACB complex $[4,5]$.

Currently, five Acinetobacter species associated with human diseases (A. baumannii, A. pittii, A. nosocomialis, A. seifertti and $A$. dijkshoorniae) and one environmental Acinetobacter species (A. calcoaceticus) are very closely related and display similar phenotypic and biochemical properties; therefore, it is difficult to distinguish them at the individual species level [6]. The accurate identification of the clinically important members of this group is only possible by molecular methods. Among the ACB complex, A. baumannii is the most important clinically 
relevant species, responsible for causing $80 \%$ of infections $[7,8]$. A. pittii and A. nosocomialis are also considered clinically important and have been implicated in causing community and hospital-acquired infections $[4,5,8]$. The two novel species mentioned previously ( $A$. seifertii and $A$. dijkshoorniae) were also isolated from human clinical specimens $[4,5]$. A calcoaceticus is considered to be a nonpathogenic environmental organism isolated from soil and is rarely involved in causing diseases [4,5].

Acinetobacter species were found to be associated with increased mortality [9]. The ACB complex has emerged as a high priority nosocomial pathogen, especially in intensive care units, thereby posing a challenge to infection management practices [10].

However, identification of Acinetobacter to the species level remains complicated. Clear differentiation among various Acinetobacter species using standard biochemical methods and automated systems are challenging, thus leading to inaccurate identification [9]. Although various molecular methods are available, they are not regularly employed in routine diagnostic laboratories [11].

Accurate identification of Acinetobacter is essential as these nosocomial pathogens, A. baumannii, A. pittii and A. nosocomialis, differ in their biological and pathological characteristics [12,13]. In addition, differences have also been observed among these pathogens in their ability to colonize the skin, antimicrobial susceptibility and mechanisms of antimicrobial resistance $[12,13]$. A. baumannii is more predominantly isolated from patients with poor outcome and increased resistance to antimicrobial agents when compared with other pathogens within the ACB complex [12,13]. A. baumannii is responsible for causing bacteremia, ventilator-associated pneumonia, urinary tract infections and skin and soft tissue infections. In 2017, Liu et al. reported A. baumannii to have a high mortality rate, of 29.8$36.9 \%$ [14]. Jain et al. reported that $A$. pittii as the most frequently isolated species with a lower rate of mortality in children [9]. Recent studies from other countries have shown that $A$. pittii and $A$. nosocomialis were responsible for $29 \%$ of ACB complex bacteremia in the USA, $24-45 \%$ in Taiwan, $50 \%$ in Korea and $66 \%$ in Norway [14].

A study by Lee $e t$ al. in 2013 clearly demonstrates important differences in the clinical features and outcome of pneumonia caused by $A$. baumannii and $A$. nosocomialis [15]. Patients infected with $A$. baumannii were more likely to have severe illness, increased antimicrobial resistance and higher mortality when compared with $A$. nosocomialis, supporting the concept that the pneumonia caused by $A$. baumannii and $A$. nosocomialis should be considered as two different clinical entities [15].

Therefore, it is important to have species-level identification of the ACB complex due to differences in epidemiology, antimicrobial resistance patterns and clinical outcomes, and reporting of infection from the ACB complex without further speciation may be considered inadequate, leading to inferior clinical care and inappropriate use of antibiotics [16].

\section{Role of phenotypic methods}

The identification scheme for Acinetobacter species, first proposed in 1986 by Bouvet and Grimont, was based on 28 phenotypic tests - physiological, nutritional and enzymatic tests [17]. Further refinement came in 1987, which includes growth at different temperatures $\left(37,41\right.$ and $\left.44^{\circ} \mathrm{C}\right)$, gelatin hydrolysis, assimilation of 14 carbon sources and acid production from glucose [18]. Based on the growth characteristics of Acinetobacter spp. at different temperatures, the scheme facilitated the differentiation of most clinically relevant isolates as A. baumannii, $A$. nosocomialis, A. pitti and A. calcoaceticus. [18,19].

The species-level identification of Acinetobacter is not consistent and reliable with the existing phenotypic methods [6]. Recently, the extension of the Bouvet and Grimont phenotypic system was proposed by Nemec, and includes growth at different temperatures $\left(32,35,41\right.$ and $\left.44^{\circ} \mathrm{C}\right)$, glucose acidification, hemolysis, gelatinase production and growth on 36 single carbon and energy sources (http://apps.szu.cz/anemec/anemec.htm). A list of phenotypic tests for the identification of ACB complex is detailed in Table 1.

\section{Role of automated bacterial identification systems}

Various commercial automated systems for identification are available and widely used in clinical microbiology laboratories [20,21]. These automated systems are based on different technologies such as measurement of growth of the microorganisms using biochemical or physical parameters, $\mathrm{CO}_{2}$ production, color, turbidity, changes in fluorescence due to microbial consumption of biochemical and carbohydrate substrates in media, production or consumption of heat, changes in pressure due to microbial consumption and bacterial detection by viability staining and metabolic activity [20,21]. The commonly used automated commercial systems for the species-level identification 
Table 1. Phenotypic characterization of species within Acinetobacter calcoaceticus-Acinetobacter baumannii complex.

\begin{tabular}{|c|c|c|c|c|c|c|}
\hline Characteristic & $\begin{array}{l}\text { Acinetobacter } \\
\text { calcoaceticus [3] }\end{array}$ & $\begin{array}{l}\text { Acinetobacter } \\
\text { baumannii [3] }\end{array}$ & $\begin{array}{l}\text { Acinetobacter } \\
\text { pittii [3] }\end{array}$ & $\begin{array}{l}\text { Acinetobacter } \\
\text { nosocomialis [3] }\end{array}$ & $\begin{array}{l}\text { Acinetobacter } \\
\text { seifertii [4] }\end{array}$ & $\begin{array}{l}\text { Acinetobacter } \\
\text { dijkshoorniae [5] }\end{array}$ \\
\hline \multicolumn{7}{|l|}{ Assimilation of: } \\
\hline$\beta$-alanine & $\mathrm{V}(-)$ & + & $V(+)$ & $\mathrm{V}(+)$ & $\mathrm{V}(+)$ & + \\
\hline L-arabinose & $V(-)$ & $V(+)$ & $V(+)$ & + & - & + \\
\hline Gentisate & - & $\mathrm{V}(-)$ & $V(-)$ & $V(-)$ & $\mathrm{V}(+)$ & + \\
\hline Leucine & V (-) & $V(+)$ & $V(+)$ & $V(+)$ & $\mathrm{V}(+)$ & + \\
\hline Malate & $\mathrm{D}$ & $V(+)$ & $V(+)$ & + & $\mathrm{V}(+)$ & + \\
\hline Ornithine & + & $\mathrm{V}(-)$ & $V(+)$ & $V(+)$ & $\mathrm{V}(+)$ & $V(+)$ \\
\hline Putrescine & + & & & & & \\
\hline Tartrate & $\mathrm{V}(-)$ & $\mathrm{V}(-)$ & $V(+)$ & - & $\mathrm{V}(+)$ & $V(+)$ \\
\hline Trigonelline & $\vee(-)$ & $V(+)$ & $V(+)$ & $V(-)$ & - & $V(+)$ \\
\hline
\end{tabular}

D: Doubtful; $\vee(+)$ : Variable, some strains are positive; $\vee(-)$ : Variable, some strains are negative.

of Acinetobacter in the clinical laboratories includes API 20NE, VITEK 2, Phoenix, Biolog, MicroScan WalkAway and Accelerate Pheno ${ }^{\top M}$ system [18].

The API $20 \mathrm{NE}$ is available with microtubes and has dehydrated media and substrates. The end products of the test are detected by an indicator or addition of reagents. The API 20NE, manual method from bioMerieux compared with conventional biochemicals in the identification of 198 isolates has reported an overall agreement of $92 \%[19,20]$. With the updated database of API 20NE (version 5.1), Bernards et al. achieved $87 \%$ accurate identification [2]. The Biolog system for automated identification of bacteria is based on the redox system [22]. Bernard and his colleagues compared strain groupings of Acinetobacter by Biolog cluster analysis with DNA-DNA hybridization $(\mathrm{DDH})$ studies and found $84.5 \%$ of the strains were correctly identified at the genus level by the Biolog system [23].

The BD Phoenix system involves the use of an oxidation-reduction indicator and turbidometric growth detection, whereas the MicroScan WalkAway system uses photometer or fluorometer to determine the growth development. The VITEK 2 system utilizes the growth based technology for microbial identification. Wang and colleagues have reported that VITEK MS method was able to accurately identify three species (A. junii, A. hemolyticus and A. johnsonii) but failed to discriminate the species within the ACB complex [24]. Such results are expected because the clinical identification system depends on the database already exists in the automated systems that are not comprehensive [18]. In addition, the substrates used for species identification have not been designed specifically for the identification of Acinetobacter [18].

Other the US FDA-cleared automated identification systems designed to identify the organisms directly from positive blood culture bottles include the Verigene system (Nanosphere) gram negative blood culture (BC-GN) nucleic acid tests and the FilmArray blood culture identification panel (Biofire diagnostics) [25,26]. The Verigene system involves purification of nucleic acids and hybridization to oligonucleotide gold nanoparticles on microarray [21]. In a study by Kim et al., Gram-negative bacteria were cultured from 150 samples, of which 22 were Acinetobacter spp and all were accurately identified by the Verigene [26]. The filmArray identification system involves extraction of DNA followed by PCR and melt curve analysis [21]. Phenotypically, A. baumannii was identified as a member of the ACB complex followed by bidirectional sequencing of the 16S rRNA gene. After comparison, the FilmArray blood culture identification detected all the 51 culture isolates as $A$. baumannii [27].

The Accelerate Pheno system, a fully automated system, has the capability of performing identification and antimicrobial susceptibility testing directly from positive blood cultures [28]. The Accelerate Pheno system combines gel electrofiltration and a broad panel of fluorescence in situ hybridization for bacterial identification and 
morphokinetic cellular analysis, which measures the response of individual cells and colonies to antibiotics over time using time-lapse imaging for antimicrobial susceptibility testing [29]. The overall identification performance has been reported with the sensitivity level of $98.6 \%$ and specificity of $99.7 \%$ with a positive predictive value and a negative predictive value of $100 \%$ for $A$. baumannii [29].

Snyder and colleagues have compared the BD Phoenix system with MicroScan WalkAway and showed 100\% concordance for the identification (ID) of nonfermentative Gram-negative bacilli (NFGNB) [30].

\section{Molecular identification of Acinetobacter species}

For the molecular identification of Acinetobacter species, several methods have been developed, studied and shown to be adequate. This includes DNA-DNA hybridization (DDH), DNA sequence-based analysis and restriction analysis of PCR amplified DNA sequences (PCR-restriction fragment length polymorphism [PCR-RFLP]). The following sections briefly describe each of the methods.

\section{DNA-DNA hybridization}

$\mathrm{DDH}$ is a gold standard method where the whole genome of the organisms is compared for delineation of bacterial species. Johnson and colleagues assigned Acinetobacter strains to five distinct DNA groups with four strains ungrouped [31]. Bouvet and Grimont distinguished 12 genospecies [32]. Bouvet and Jeanjean described genomic species 13-17 [33]. A parallel study to Bouvet and Jeanjean describing three new genomic species 13-15 was reported by Tjernberg and colleagues [34]. In 1988, the genomic sp. 12 was described as A. radioresistens [35].

Although DDH has been considered a gold standard for bacterial species differentiation over the past 50 years, due to several disadvantages like a labor-intensive and error prone nature, genotype-based technology was needed [36]. As whole-genome sequencing is available, numerous overall genome relatedness indices (OGRI) were developed to replace DDH [37].

The OGRI is digital DDH-like similarity indices based on computational comparisons of two genome sequences [36]. Various OGRI are available, including average nucleotide identity (ANI), genome BLAST distance phylogeny and maximal unique matches index [36]. Among these, ANI is most widely used and considered as the gold standard method for species demarcation [36,37]. Generally, ANI values of 95-96\% are accepted for species delineation, corresponding to the traditional $70 \% \mathrm{DDH}$ threshold [36]. To calculate the reciprocal ANI values, a new algorithm called OrthoANI (ANI by orthology) is available [37].

Chan et al., using the genus Acinetobacter, examined whether bacterial taxonomy could rely upon genome-based sequence data using ANI [38]. For example, $A$. nosocomialis NCTC 10304 exhibits ANI values more than 95\% with 14 sequenced $A$. baumannii, thereby confirming the designation as $A$. baumannii NCTC 10304. Similarly, $A$. calcoaceticus PHEA-2 was designated as $A$. pittii PHEA-2 [38]. Since then various studies have used ANI for species delineation of the genus Acinetobacter [4,5,39,40].

\section{DNA sequence-based analysis}

DNA sequence-based analysis is used to understand the relatedness of organisms from their DNA sequence similarity. Various DNA sequences like 16S rRNA, rpoB, 16S-23S intergenic spacer (ITS) have been studied in classification and identification of Acinetobacter.

\section{5 rRNA \& rpoB sequencing}

$16 \mathrm{~S}$ rRNA sequencing is the most commonly used gold standard technique for unknown identification of bacterial species [41]. The $16 \mathrm{~S}$ rRNA gene of bacteria contains nine hypervariable regions that show sequence diversity among the different bacterial species and are therefore used for species-level identification [42]. The V8 hypervariable region of 16S rRNA is useful in the identification of Acinetobacter at the genus level [43]. Misbah et al. amplified $1500 \mathrm{bp}$ 16S rRNA region using three primer pairs to identify clinical isolates of Acinetobacter species and all were identified as $A$. baumannii [44]. The intraspecies similarity of $16 \mathrm{~S} \mathrm{rRNA}$ for $A$. baumannii is $99.9-100 \%$, A. nosocomialis 99.7-100\%, A. pittii 99.8-100\%, A. calcoaceticus 97.6-99.3\%, A. seifertii 97.4-97.8\%, A. dijkshoorniae 99-100\%. The interspecies similarity of $16 \mathrm{~S} \mathrm{rRNA}$ of $A$. baumannii with $A$. nosocomialis, $A$. pittii, A. calcoaceticus, A. seifertii and $A$. dijkshoorniae were 97.2, 97.1, 96.9, 98.5 and 97.1\%, respectively. Although 16S rRNA sequencing was reported to be polymorphic for the identification of other bacterial genera, it is not effective in differentiating Acinetobacter at the species level due to its extremely low polymorphic nature of the variable region [45]. Due to this limitation, protein encoding genes like $r p o B, \operatorname{gyr} B$ and $r e c A$, which evolve much faster, were investigated as 
diagnostic biomarkers. The $r p o B$ gene is 4089 base pair (bp) long and only one copy is present [45]. The rpoB gene has four variable regions which includes two polymorphic regions and two flanking spacers enclosed by conserved sequences [45]. The intraspecies similarity of $r p o B$ for $A$. baumannii was $98.7-100 \%$, A. nosocomialis $98.7-100 \%$, A. pittii $98.6-100 \%$, A. seifertii $98.5-100 \%$, A dijkshoorniae $98.6-100 \%$ and A. calcoaceticus $97.7-99.7 \%$ [5]. The interspecies similarity of rpoB for $A$. baumannii with $A$. pittii, A.nosocomialis, A. calcoaceticus, A. seifertii and $A$, dijkshoorniae were 92-93\%, 94-95\%, 91-92\%, 94-95\% and 93-94\%, respectively [5].

Recently, Lee and colleagues reported, 93.4 and $98.2 \%$ accuracy for the species-level identification of Acinetobacter by $16 \mathrm{~S}$ rRNA and $r p o B$ sequencing, respectively [46]. Similarly, Khosravi's group revealed that $16 \mathrm{~S}$ rRNA sequencing of $A$. grimontii and $A$. junii, was ambiguous due to high similarity between these two species, whereas the rpoB gene sequencing showed 100 and $99 \%$ similarity to $A$. grimontii and $A$. junii, respectively [47]. Initially, Vaneechoutte $e t$ al. proposed that $A$. grimontii as a heterotypic synonym of $A$. junii by DDH and AFLP [48]. Wang et al. reported that the similarity of $r p o B$ sequences of $A$. grimontii and $A$. junii is greater than $99 \%$, thereby suggesting their combination into one species [49].

La Scola's group investigated the efficacy of $r p o B$ gene sequencing for the differentiation and identification of 17 reference strains of Acinetobacter spp. and seven unnamed genome species. The percentage similarities of $r p o B$ gene sequences of different strains varied between 83 and 99\% [45]. Furthermore, Gundi and colleague studied the use of partial $r p o B$ sequence analysis (PRBS) on a collection of Acinetobacter spp. by analyzing residues spanning the 350 base pair (bp) hypervariable zone of the $r p o B$ gene [50]. With partial $r p o B$ sequence analysis, it was found that while interspecies similarity were well separated (ranging from 88.3 to $96.9 \%$ ), the intraspecies similarity of the $r p o B$ gene sequences was found to be $98-100 \%$ for $A$. baumannii, $98-100 \%$ for $A$. nosocomialis, $98-100 \%$ for A. pitti and $98.6-100 \%$ for $A$. calcoaceticus [50].

In summary, while $16 \mathrm{~S}$ rRNA sequencing is highly reliable at the genus level, it has poor discriminatory ability at the species level. In contrast, rpoB genes among Acinetobacter spp. are highly variable and considered to be appropriate for species identification [49].

\section{Restriction analysis of PCR amplified DNA sequences}

This method involves amplification and restriction of conserved gene sequences using various restriction enzymes. Several gene sequences such as 16S rRNA, 16S-23S ITS and recA are widely used [51].

In the amplified ribosomal DNA restriction analysis (ARDRA) method, amplification of the 16S rRNA gene followed by RFLP using different restriction enzymes enabled identification of all Acinetobacter species except A. haemolyticus (genomic species 4) and A. johnsonii (genomic species 7) [1]. However, when Nowak et al. used the 16S-23S ITS region, identical PCR and RFLP patterns were observed [52]. Multiplex PCR targeting recA and $16 \mathrm{~S}$ rDNA genes followed by RFLP yielded reliable identification of Acinetobacter geno species five, seven and ten including the closely related species within ACB complex [53].

Although the above methods are helpful in understanding the clinical relevance and epidemiology of Acinetobacter species, they are too laborious for day-to-day application in the laboratory or field setting. As these techniques involve skilled labor, they are restricted only to reference laboratories.

\section{Sequence-based analysis of other gene targets}

The other sequence targets available for the identification and discrimination of Acinetobacter species are summarized in Table 2. A multiplex PCR targeting the recA and 16S-23S rRNA ITS region for the identification of Acinetobacter species within the ACB complex was developed using 22 reference strains and 138 clinical isolates of Acinetobacter spp. The multiplex PCR was found to be $100 \%$ accurate in identification of Acinetobacter at the species level, which was later confirmed by sequence analysis [54]. In 2013, Soo and colleagues developed a loop-mediated isothermal amplification (LAMP) method for identification of $A$. baumannii using the 16S-23S rRNA ITS region. The sensitivity of the LAMP assay was found to be 100-fold superior to the simple multiplex PCR method in identifying Acinetobacter species [55]. However, the main drawback is that the LAMP method has not been studied on different clinical isolates.

Another reliable marker for identification of $A$. baumannii is the bla $\mathrm{OXA}-51$-like gene, which is intrinsic to A. baumannii. The utility of bla $\mathrm{OXA}_{-51}$-like gene-specific PCR has been studied by Turton and colleagues on 106 A. baumannii isolates and 23 different Acinetobacter spp, which were previously identified by amplified $16 S$ rDNA restriction analysis (ARDRA). All 106 A. baumannii clinical isolates, as well as the ATCC 19606 strain, flagged positive for bla $\mathrm{OXA}-51$-like gene, whereas isolates that showed negative were identified as another species of 
Table 2. Various PCR-based targets for identification of Acinetobacter spp.

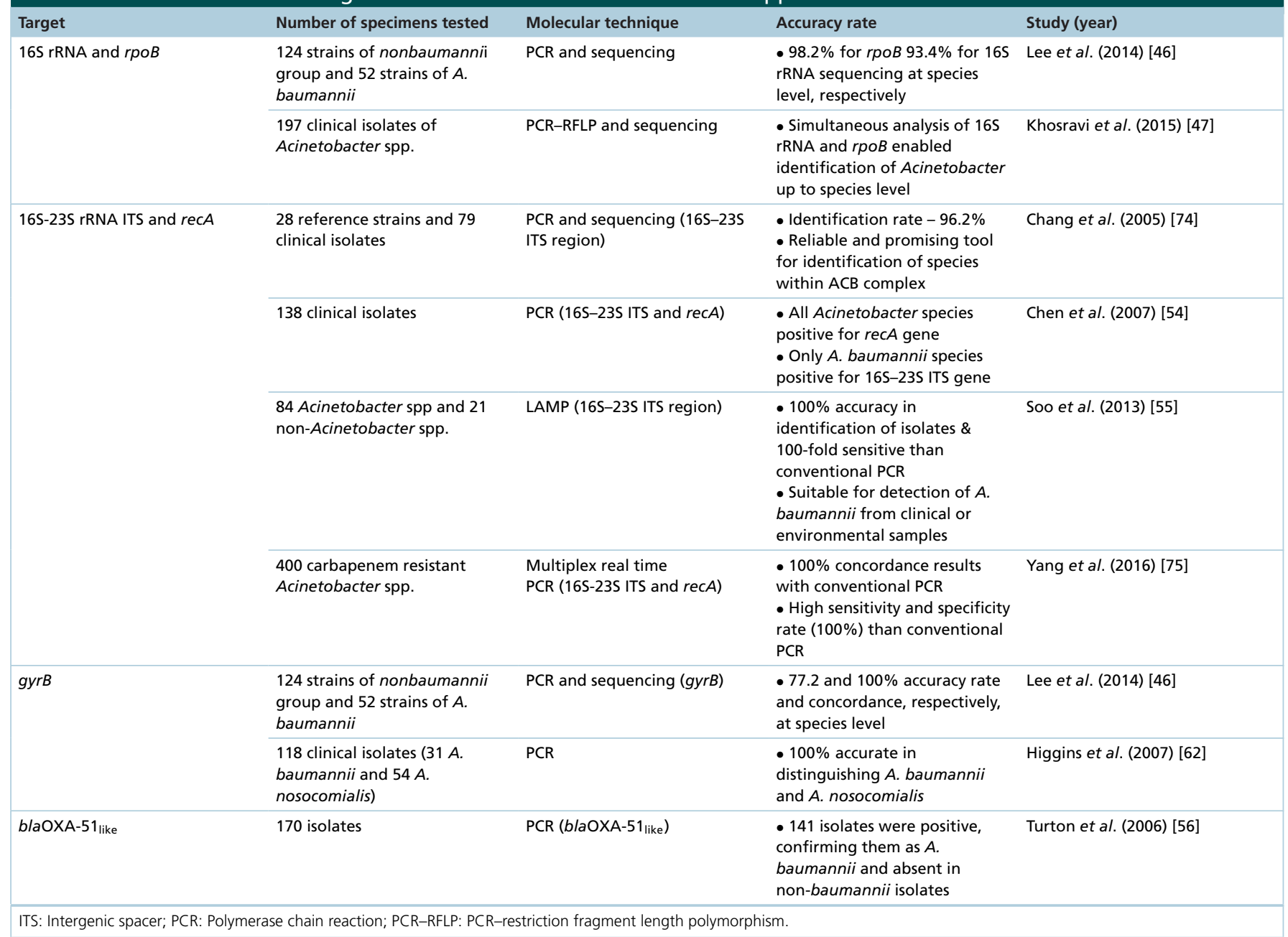

Acinetobacter. So far, 95 variants of bla $\mathrm{OXA}-51$ like gene have been described and the possible limitation of this PCR method could be due to its inability to detect all the described variants of the bla OXA-51 gene [56,57].

However, false-positive and false-negative results for detection of the bla $a_{\mathrm{OXA}-51}$ gene were reported by a few studies, as mentioned below. False-positive results were observed by Chen's group in two different studies involving 676 and 74 isolates of non-baumannii Acinetobacter species, which showed 0.9 and $5.5 \%$ prevalence of the bla $a_{\mathrm{OXA}-51}$-like

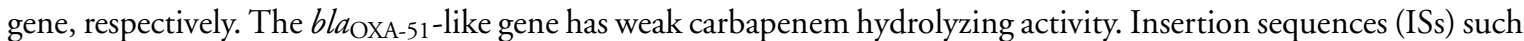
as ISAbal could be inserted upstream, leading to overexpression of the blaOXA-51-like genes [58]. It was reported that

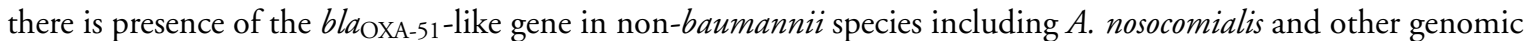
species close to $A$. nosocomialis. All these isolates contain the upstream insertion of ISAbal on plasmids, which might have been transferred from $A$. baumannii by the horizontal gene transfer method [59].

The false negative issue was also observed during an outbreak situation, where approximately 14 isolates generated a 1.7-kb PCR product instead of $353 \mathrm{bp}$ for this marker. Sequencing of the 1.7-kb PCR band revealed disruption

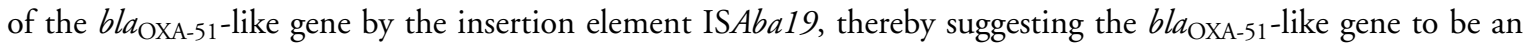
unsuitable molecular marker for $A$. baumannii identification [60]. A separate study also reported disruption of the

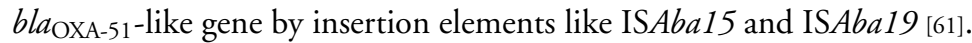

Notably, presence of such plasmid-borne insertion elements along with bla $a_{\text {OXA-51-like gene confers high level }}$ carbapenem resistance, thereby limiting the use of bla $a_{\mathrm{OXA}-51-l i k e}$ gene as a target for differentiating $A$. baumannii from other Acinetobacter species. Therefore, it is essential to include additional gene targets like rpo $B$ sequencing

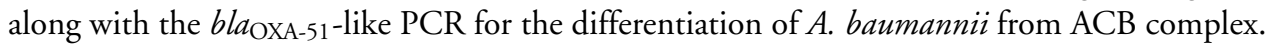


Multiplex PCR based on species-specific genes is considered simple, rapid and reliable for identification of different species within the ACB complex. This multiplex PCR is robust, reproducible and cost effective and yields results in less than $2 \mathrm{~h}$. A multiplex PCR based on $\operatorname{gyr} B$ primers was proposed for the identification of $A$. baumannii and $A$. nosocomialis [62]. This multiplex PCR was expanded to include identification of $A$. pittii and A. calcoaceticus [63]. A total of 495 clinical isolates of Acinetobacter were subjected to gyrB multiplex PCR and VITEK2 analysis. The accuracy rates were found to be 90.5 and $76.6 \%$ for $g y r B$ multiplex PCR and VITEK2, respectively [46]. The gyrB multiplex PCR showed $100 \%$ concordance in identifying species within the ACB complex whereas VITEK2 was able to identify only $A$. baumannii and failed to identify other species within the ACB complex [46].

Although various target regions like $\operatorname{rec} A$ and $\operatorname{gyr} B$ are available for the identification and differentiation of Acinetobacter species within the ACB complex, none of them has been validated for the recently included novel species $A$. seifertii and $A$. dijkshoorniae, which can be considered as a limitation.

\section{Role of MALDI-TOF}

Matrix-assisted laser desorption ionization-time of flight mass spectrometry (MALDI-TOF) is increasingly used in diagnostic microbiology for the routine identification of bacteria at the genus, species or subspecies level [64,65]. Generally, MALDI-TOF is prepared by coating the sample with an energy absorbent solution called matrix. The matrix is crystallized while drying and the sample within the matrix is also crystallized [66]. The sample is then subjected to a laser beam. Desorption and ionization with the laser beam produces protonated ions from analytes such as protein in the sample, which are accelerated and separated from each other based on their $\mathrm{m} / \mathrm{z}$ [66,67]. Afterward, the charged analytes are detected and measured using a TOF analyzer. A characteristic peptide-mass-fingerprint (PMF) is generated for analytes in the sample based on the TOF information [66,67]. Microbial identification by MALDI-TOF is performed by comparing the PMF of unknown organism with PMFs available in the database [66].

Bacterial cultures subjected to ethanol-formic acid extraction as well as directly from bacterial colonies were used for identification [68]. Table 3 summarizes various studies carried out for the identification of Acinetobacter spp. using MALDI-TOF.

Espinal's group tested the previously characterized 60 Acinetobacter isolates (18 A.nosocomialis, 17 A. pittii and 18 A. baumannii) and seven reference strains of different Acinetobacter species using MALDI-TOF with Bruker database. It was found that $85 \%(\mathrm{n}=6)$ accuracy in identification at species level for the reference strains. Specieslevel identification was accurate and achieved for all A. baumannii $(100 \%, \mathrm{n}=18)$ isolates and all $A$. pittii $(94 \%$, $\mathrm{n}=17$ ), except one. None of the $A$. nosocomialis were identified by MALDI-TOF as the Bruker database lacks a signature specific for $A$. nosocomialis. A set of reference spectra was created using three strains each of $A$. baumannii, A. pittii and $A$. nosocomialis to supplement the Bruker database. Reanalysis of spectra from all 60 isolates using a local database incorporated to MALDI-TOF was performed with reference signatures of $A$. nosocomialis, which provided unequivocal identification for all the tested strains [69].

Sedo et al. found an inability of MALDI-TOF to discriminate between A. baumannii and A. nosocomialis within the closely related ACB complex. In their study with a taxonomically clearly defined set of 105 strains representing the four species of the ACB complex, they evaluated a protocol with change in the matrix composition without changing the other steps of the sample processing. With the modified protocol, differentiation between A. baumannii and $A$. nosocomialis was improved and 13 A. nosocomialis strains which were wrongly assigned were correctly identified [70].

Hsueh et al. evaluated the ability of MALDI-TOF (Bruker Biotyper) in the correct identification of species within the ACB complex as well as species other than the ACB complex. The overall accurate identification rate of species within the ACB complex was only $85.3 \%$.

Although the current database included $A$. nosocomialis, the correct identification rate was $72.4 \%$. Additionally, accurate identification of non-ACB complex species like A. junii, A. ursingii, A. johnsonii and $A$. radioresistens was achieved using the Bruker Biotyper [71].

Kishii and colleagues evaluated MALDI-TOF for the identification of Acinetobacter to the species level by comparison with sequence analysis of $r p o B$ using 123 isolates of Acinetobacter species [72]. MALDI-TOF identified $86.2 \%$ of Acinetobacter isolates to species level and $13 \%$ of the isolates to genus level. With rpoB sequence analysis, $84 \%$ were confirmed to the correct species level and discordant results were obtained for $16 \%$ isolates. The 
Table 3. Various studies using MALDI-TOF for the identification of Acinetobacter spp.

\begin{tabular}{|c|c|c|c|c|}
\hline $\begin{array}{l}\text { Number of specimens } \\
\text { tested }\end{array}$ & $\begin{array}{l}\text { Species identification } \\
\text { using reference method } \\
\text { (rpoB sequencing and } \\
\text { bla } \\
\text { OXA-51-like) }\end{array}$ & $\begin{array}{l}\text { Species identified using } \\
\text { MALDI-TOF }\end{array}$ & Accuracy rate & Study (year) \\
\hline 109 & $\begin{array}{l}\text { A. baumannii - } 85 \\
\text { (blaoxA-51-like) } \\
\text { A. genospecies } 3-8, A \text {. } \\
\text { berezinae }-7 \\
\text { A. calcoaceticus - } 2 \\
\text { A. genospecies 14BJ - } 1 \\
\text { A. genospecies 13TU - } 3 \\
\text { A. junii-1, } \\
\text { A. soli }-1 \\
\text { A. beijerinkii-1 } 1\end{array}$ & $\begin{array}{l}\text { A. baumannii }-87, \\
\text { A. genospecies } 3-19 \text {, } \\
\text { A. junii }-1, \text { A. baylyi }-1 \\
\text { A. tjernbergiae }-1\end{array}$ & $\begin{array}{l}\text { MALDI-TOF - at species } \\
\text { level: } \\
\text { Accurate identification - } \\
51.6 \% \\
\text { Misidentification - } 48.4 \% \\
\text { For } \text { A. baumannii: } \\
\text { Concordance }-77.8 \% \\
\text { Sensitivity - } 100 \% \\
\text { Specificity }-91.7 \%\end{array}$ & $\begin{array}{l}\text { Álvarez-Buylla et al. } \\
\text { (2012) [76] }\end{array}$ \\
\hline 60 & $\begin{array}{l}\text { A. baumannii }-100 \% \\
\text { (blaoxA-51-like) }\end{array}$ & $\begin{array}{l}\text { A. baumannii-18, } \\
\text { A. nosocomialis }-18 \\
\text { A. pitti- } 17 \\
\text { Reference strains }-7\end{array}$ & $\begin{array}{l}98 \% \text { of all isolates were } \\
\text { correctly identified up to } \\
\text { appropriate species level }\end{array}$ & Espinal et al. (2012) [69] \\
\hline $\begin{array}{l}409 \\
\text { (A. baumannii - } 24 \\
\text { Non-baumannii species - } \\
\text { 385) }\end{array}$ & $\begin{array}{l}\text { A. pitti/ A. calcoaceticus - } \\
\text { 189 } \\
\text { A. oleivorans/A. } \\
\text { calcoaceticus - } 6 \\
\text { A. nosocomialis - } 134 \\
\text { A. genomic species } \\
33 Y U-11 \\
\text { A. baumannii- } 24 \\
\text { A. haemolyticus - } 2 \\
\text { A. johnsonii/A. Iwoffii - } 4 \\
\text { A. junii/A. grimontii- } 25 \\
\text { A. soli-9 } \\
\text { A. bereziniae - } 4 \\
\text { A. ursingii - } 1\end{array}$ & $\begin{array}{l}\text { ACB complex }-370 \\
\text { A. hemolyticus }-2 \\
\text { A. johnsonii- } 4 \\
\text { A. junii }-25 \\
\text { Non -Acinetobacter spp. } \\
-7 \\
\text { Escherichia coli - } 1\end{array}$ & $\begin{array}{l}\text { Identical results obtained } \\
\text { with MALDI-TOF for } A \text {. } \\
\text { hemolyticus, A. johnsonii, } \\
\text { and A. junii as rpoB } \\
\text { sequencing } \\
\text { MALDI-TOF was not able } \\
\text { to discriminate the } \\
\text { species within the ACB } \\
\text { complex }\end{array}$ & Wang et al. (2014) [24] \\
\hline 176 & & $\begin{array}{l}\text { A. baumannii- } 101 \\
\text { A. nosocomialis }-43 \\
\text { A. pitti-10 } \\
\text { A. genomic } \\
\text { species } 13 \mathrm{BJ} / 14 \mathrm{TU}-10 \\
\text { A. ursingii - } 4 \\
\text { A. soli- } 4 \\
\text { A. bereziniae }-2 \\
\text { A. junii - } 2\end{array}$ & $\begin{array}{l}\text { A. baumannii- } 101 \\
\text { A. nosocomialis - } 43 \\
\text { A. pitti-9 } \\
\text { A. ursingii- } 4 \\
\text { A. junii- } 1 \\
\text { Mis-identification - } 18\end{array}$ & $\begin{array}{l}99.4 \% \text { of the isolates Lee et al. (2015) [77] } \\
\text { within the ACB complex } \\
\text { and } 27.3 \% \text { of the isolates } \\
\text { among the } \\
\text { nonbaumannii group } \\
\text { were correctly identified } \\
\text { with MALDI-TOF } \\
\text { Correct ID rate }-90.3 \% \\
\text { Mis-ID rate }-0.6 \% \\
\text { No ID rate }-9.1 \%\end{array}$ \\
\hline $\begin{array}{l}\text { Panel of ACB complex } \\
\text { collection - } 91 \text { for } \\
\text { confirmation ofupdated } \\
\text { database - SARAMIS } \\
\text { (spectral archive and } \\
\text { microbial identification } \\
\text { system) }\end{array}$ & $\begin{array}{l}\text { A. pitti }-50 \\
\text { A. baumannii- } 40 \\
\text { A. nosocomialis }-1\end{array}$ & $\begin{array}{l}\text { A. pitti }-50 \\
\text { A. baumannii- } 40 \\
\text { A. nosocomialis }-1\end{array}$ & $\begin{array}{l}90 \% \text { of } A . \text { baumannii and } \\
62 \% \text { of } A . \text { pittii isolates } \\
\text { were accurately identified }\end{array}$ & $\begin{array}{l}\text { Pailhories et al. } \\
\text { (2015) [78] }\end{array}$ \\
\hline $\begin{array}{l}\text { Default database - } 212 \\
\text { isolates } \\
\text { Improved database - } 517 \\
\text { isolates }\end{array}$ & - & - & $\begin{array}{l}\text { Default database }-69.8 \% \\
\text { concordance } \\
\text { Improved database - } \\
100 \% \text { concordance }\end{array}$ & Jeong et al. (2016) [79] \\
\hline
\end{tabular}


$r p o B$ sequencing, which is better for species delineation, was able to assign 123 isolates to 13 species whereas MALDI-TOF assigns only to 9 species indicating the less discriminatory power of MALDI-TOF [72].

MALDI-TOF combined with chemometric tools like partial least squares discriminant analysis and hierarchical cluster analysis was reported to have (100\%) accuracy in the identification within the ACB complex [73]. Overall the diagnostic efficiency of MALDI-TOF from different studies, showed $100 \%$ sensitivity, $91.7-99 \%$ specificity and $70-100 \%$ concordance with other methods for identification of $A$. baumannii.

MALDI-TOF technique was unable to identify the two novel species, $A$. seifertii and $A$. dijkshoorniae and several studies have shown that the estimated identification rate for $A$. nosocomialis range at $70 \%$ suggesting to update and improve the database for accurate identification of species within the ACB complex [68]. A total of 78 Acinetobacter isolates representing A. baumannii, A. nosocomialis, A. pittii, A. dijkshoorniae and A. seifertii were used to calculate the species-specific average spectra. Mean spectra derived from $A$. seifertii, A. dijkshoorniae and $A$. nososcomialis were added to the database [68]. Finally, when spectra from all the 78 isolates were analyzed using the custom database which included both the mean spectra from all the Acinetobacter species in the default taxonomy with the new reference signatures of $A$. nosocomialis, A. seifertii and A. dijkshoorniae allowed the identification of Acinetobacter to the correct species level within the ACB complex [68].

Variations in the sensitivity and specificity in identifying ACB complex by MALDI-TOF have been reported [68]. This is due to the methodological differences followed in each of the study. High rates of $>98 \%$ of sensitivity and specificity were obtained when the bacterial colonies were subjected to a modified extraction protocol using formic acid and acetonitrile, compared with the conventional method which yielded a sensitivity and specificity of 91 and $98 \%$, respectively [68,69]. Though both the protocols have used total mass for identification, the methodological variation of sample preparation impacts on the overall sensitivity and specificity rates.

\section{Conclusion}

Species within the ACB complex are difficult to distinguish. The differences in clinical relevance of the ACB complex and their antimicrobial susceptibility profile warrant species-level identification. The traditional phenotypic methods such as culture and biochemical identification are slow, unreliable and less efficient at differentiating the most clinically important $A$. baumannii within the ACB complex. PCR targets such as the bla OXA-51-like gene, $16 \mathrm{~S}-_{-}$ $23 \mathrm{~S}$ rRNA ITS, recA and $g y r B$; and PCR-RFLP of $16 \mathrm{~S}$ rRNA, 16S-23S ITS and recA were considered as suitable markers for Acinetobacter speciation. However, these methods are mostly performed by reference laboratories. With an accurate database, MALDI-TOF can be helpful for species-level identification. To conclude, molecular characterization using $b l_{\mathrm{OXA}-51}$-like PCR together with $r p o B$ sequencing has high discriminatory power over the conventional methods for Acinetobacter species identification, especially within the ACB complex.

\section{Future perspective}

Identification of Acinetobacter species within the ACB complex is still challenging. Currently, available phenotypic methods are insufficient. Although various commercially available automated identification systems are widely used in clinical laboratories, such systems are still not effective for identification. Incorporation of more diverse mass spectra of different species within the ACB complex will improve the MALDI-TOF database, which eventually will be useful in accurate identification of the ACB complex at the species level. For molecular characterization, a single gene target as a biomarker is not sufficient for accurate identification of ACB complex. Whole-genome sequencing can be considered as a promising tool; however, its usage is limited in lower resource settings. Furthermore, comparative genomic studies will help us to identify the species-specific targets that can be used as a marker for molecular identification.

\section{Financial \& competing interests disclosure}

The authors have no relevant affiliations or financial involvement with any organization or entity with a financial interest in or financial conflict with the subject matter or materials discussed in the manuscript. This includes employment, consultancies, honoraria, stock ownership or options, expert testimony, grants or patents received or pending, or royalties.

No writing assistance was utilized in the production of this manuscript.

\section{Author contributions}

S Vijayakumar reviewed the literature and drafted the manuscript. B Veeraraghavan and I Biswas reviewed and edited the manuscript. All authors read and approved the manuscript. 


\section{Summary points}

- Identification of Acinetobacter species within the closely related Acinetobacter calcoaceticus-Acinetobacter baumannii complex is crucial.

- The existing phenotypic methods and commercial automated systems are not reliable for the species-level identification of Acinetobacter.

- At the genus level, 16S rRNA sequencing is reliable whereas $r p o B$ sequencing is considered to be appropriate for species level identification.

- blaOXA-51-like gene is considered to be the species-specific biomarker for Acinetobacter baumannii.

- Presence of plasmid-borne insertion elements along with blaOXA-51-like gene confers carbapenem resistance, thereby limiting the use of blaOXA-51-like gene as a target for differentiating Acinetobacter baumannii from other Acinetobacter species.

- MALDI-TOF with an accurate database will be useful in the species-level identification within the A. calcoaceticus-A. baumannii complex.

Open access

This work is licensed under the Creative Commons Attribution 4.0 License. To view a copy of this license, visit http://creativecomm ons.org/licenses/by/4.0/〈0:bold $\rangle\langle/ 0$ :bold $\rangle$

\section{References}

Papers of special note have been highlighted as: $\bullet$ of interest; $\bullet \bullet$ of considerable interest.

1. Vaneechoutte M, Dijkshoorn L, Tjernberg I et al. Identification of Acinetobacter genomic species by amplified ribosomal DNA restriction analysis. J. Clin. Microbiol. 33(1), 11-15 (1995).

2. Bernards AT, Van der Toorn J, Van Boven CPA et al. Evaluation of the ability of a commercial system to identify Acinetobacter genomic species. Eur. J. Clin. Microbiol. Infect. Dis. 15(4), 303-308 (1996).

3. Alexander Nemec (2017). http://apps.szu.cz/anemec/anemec.htm

4. Nemec A, Krizova L, Maixnerova M et al. Acinetobacter seifertii sp. nov., a member of the Acinetobacter calcoaceticus-Acinetobacter baumannii complex isolated from human clinical specimens. Int. J. Syst. Evol. Microbiol. 65(3), 934-942 (2015).

- First study to describe the new species Acinetobacter seifertii within the Acinetobacter calcoaceticus-Acinetobacter baumannii complex.

5. Cosgaya C, Marí-Almirall M, Van Assche A et al. Acinetobacter dijkshoorniae sp. nov., a member of the Acinetobacter calcoaceticus-Acinetobacter baumannii complex mainly recovered from clinical samples in different countries. Int. J. Syst. Evol. Microbiol. 66(10), 4105-4111 (2016).

- First study to describe the new species Acinetobacter dijkshoorniae within the $A$. calcoaceticus-A. baumannii complex.

6. Howard A, O'Donoghue M, Feeney A et al. Acinetobacter baumannii: an emerging opportunistic pathogen. Virulence 3(3), 243-250 (2012).

7. Manchanda V, Sanchaita S, Singh NP. Multidrug resistant Acinetobacter. J. Glob. Infect. Dis. 2(3), 291 (2010).

8. Chusri S, Chongsuvivatwong V, Rivera JI et al. Clinical outcomes of hospital-acquired infection with Acinetobacter nosocomialis and Acinetobacter pittii. Antimicrob. Agents Chemother. 58(7), 4172-4179 (2014).

9. Jain AL, Harding CM, Assani K et al. Characteristics of invasive Acinetobacter species isolates recovered in a pediatric academic center. BMC Infect. Dis. 16(1), 346 (2016).

10. Chen L, Yuan J, Xu Y et al. Comparison of clinical manifestations and antibiotic resistances among three genospecies of the Acinetobacter calcoaceticus-Acinetobacter baumannii complex. PLoS ONE 13(2), e0191748 (2018).

11. Wisplinghoff $\mathrm{H}$, Paulus $\mathrm{T}$, Lugenheim $\mathrm{M}$ et al. Nosocomial bloodstream infections due to Acinetobacter baumannii, Acinetobacter pittii and Acinetobacter nosocomialis in the United States. J Infect. 64(3), 282-290 (2012).

12. Lee YT, Kuo SC, Yang SP et al. Impact of appropriate antimicrobial therapy on mortality associated with Acinetobacter baumannii bacteremia: relation to severity of infection. Clin. Infect. Dis. 55(2), 209-215 (2012).

13. Chuang YC, Sheng WH, Li SY et al. Influence of genospecies of Acinetobacter baumannii complex on clinical outcomes of patients with Acinetobacter bacteremia. Clin. Infect. Dis. 52(3), 352-360 (2011).

14. Liu YM, Lee YT, Kuo SC et al. .. Comparison between bacteremia caused by Acinetobacter pittii and Acinetobacter nosocomialis.. J. Microbiol. Immunol. Infect. 50(1), 62-67 (2017).

15. Lee YT, Kuo SC, Yang SP et al. Bacteremic nosocomial pneumonia caused by Acinetobacter baumannii and Acinetobacter nosocomialis: a single or two distinct clinical entities. Clin. Microbiol. Infect. 19(7), 640-645 (2013). 
16. Fitzpatrick MA, Ozer E, Bolon MK et al. Influence of ACB complex genospecies on clinical outcomes in a US hospital with high rates of multidrug resistance. J. Infect. 70(2), 144-152 (2015).

17. Bouvet PJ, Grimont PA. Taxonomy of the genus Acinetobacter with the recognition of Acinetobacter baumannii sp. nov., Acinetobacter haemolyticus sp. nov., Acinetobacter johnsonii sp. nov., and Acinetobacter junii sp. nov. and emended descriptions of Acinetobacter calcoaceticus and Acinetobacter lwoffi. Int. J. Syst. Evol. Microbiol. 36(2), 228-240 (1986).

18. Peleg AY, Seifert H, Paterson DL. Acinetobacter baumannii: emergence of a successful pathogen. Clin. Microbiol. Rev. 21(3), 538-582 (2008).

-• Describes an overview of microbiology, mechanisms of antibiotic resistance, epidemiology, clinical manifestations, infection control, molecular epidemiologic techniques and therapeutic strategies of Acinetobacter baumannii.

19. Molecular Typing in Bacterial Infections, Infectious Disease. de Filippis I, McKee ML, (Eds). Humana Press NY, USA (2013).

20. O'Hara CM. Manual and automated instrumentation for identification of Enterobacteriaceae and other aerobic Gram-negative bacilli. Clin. Microbiol. Rev. 18(1), 147-162 (2005).

- Gives an overview of various manual and automated identification systems.

21. Li Y, Yang X, Zhao W et al. Emerging microtechnologies and automated systems for rapid bacterial identification and antibiotic susceptibility testing. SLAS Technol. 22 (6), 585-608 (2017).

22. Stefanowicz A. The Biolog plates technique as a tool in ecological studies of microbial communities. Polish J. Environ. Stud. 15 (5), 669-676 (2006).

23. Bernards AT, Dijkshoorn L, Van der Toorn J et al. Phenotypic characterisation of Acinetobacter strains of 13 DNA-DNA hybridisation groups by means of the Biolog system. J. Med. Microbiol. 42(2), 113-119 (1995).

24. Wang J, Ruan Z, Feng Y et al. Species distribution of clinical Acinetobacter isolates revealed by different identification techniques. PLoS ONE 9(8), e104882 (2014).

25. Bhatti MM, Boonlayangoor S, Beavis KG et al. Evaluation of filmarray and verigene systems in the rapid identification of positive blood cultures. J. Clin. Microbiol. 52(9) 3433-3436 (2014).

26. Kim JS, Kang GE, Kim HS et al. Evaluation of Verigene blood culture test systems for rapid identification of positive blood cultures. Biomed. Res. Int. 1081536, 1-6 (2016).

27. Salimnia H, Fairfax MR, Lephart PR et al. Evaluation of the FilmArray ${ }^{\circledR}$ blood culture identification panel: results of a multi-center controlled trial. J. Clin. Microbiol. 54(3) 687-698 (2016).

28. Marschal M, Bachmaier J, Autenrieth I et al. Evaluation of the Accelerate Pheno ${ }^{\mathrm{TM}}$ system for fast identification and antimicrobial susceptibility testing from positive blood culture in Gram-negative bloodstream infection. J. Clin. Microbiol.55(7) 2116-2126 (2017).

29. Charnot-Katsikas A, Tesic V, Love N. Use of Accelerate Pheno ${ }^{\text {TM }}$ system for identification and antimicrobial susceptibility testing (ID/AST) of pathogens in positive blood cultures and impact on time to results and workflow. J. Clin. Microbiol. 56 pii = e01166-17 (2017).

30. Snyder JW, Munier GK, Johnson CL. Direct comparison of the BD phoenix system with the MicroScan WalkAway system for identification and antimicrobial susceptibility testing of Enterobacteriaceae and nonfermentative gram-negative organisms. J. Clin. Microbiol. 46(7), 2327-2333 (2008).

31. Johnson JL, Anderson RS, Ordal EJ. Nucleic acid homologies among oxidase-negative Moraxella species. J. Bacteriol. 101(2), 568-573 (1970).

32. Dijkshoorn L, Nemec A, In: The diversityof the genus Acinetobacter. Acinetobacter molecularmicrobiology. Gerischer U, (Eds). Norfolk, UK, CaisterAcademic Press, 1-34 (2008).

33. Bouvet PJM, Jeanjean S. Delineation of new proteolytic genomic species in the genus Acinetobacter. Res. Microbiol. 140(4), 291-299 (1989).

34. TJERNBERG I, Ursing JAN. Clinical strains of Acinetobacter classified by DNA-DNA hybridization. APMIS 97(7-12), 595-605 (1989).

35. Nishimura Y, Ino T, Iizuka H. Acinetobacter radioresistens sp. nov. isolated from cotton and soil. Int. J. Syst. Evol. Microbiol. 38(2), 209-211 (1988).

36. Kim M, Oh HS, Park SC et al. Towards a taxonomic coherence between average nucleotide identity and 16S rRNA gene sequence similarity for species demarcation of prokaryotes. Int. J. Syst. Evol. Microbiol. 64(2), 346-351 (2014).

37. Lee I, Kim YO, Park SC et al. OrthoANI: an improved algorithm and software for calculating average nucleotide identity. Int. J. Syst. Evol. Microbiol. 66(2), 1100-1103 (2016).

38. Chan JZ, Halachev MR, Loman NJ et al. Defining bacterial species in the genomic era: insights from the genus Acinetobacter. $B M C$ Microbiol. 12(1), 302 (2012).

39. Touchon M, Cury J, Yoon EJ et al. The genomic diversification of the whole Acinetobacter genus: origins, mechanisms, and consequences. Genome Biol. Evol. 6(10), 2866-2882 (2014). 
40. Montaña S, Schramm ST, Traglia GM et al. The genetic analysis of an Acinetobacter johnsonii clinical strain evidenced the presence of horizontal genetic transfer. PLoS ONE 11(8), e0161528 (2016).

41. Clarridge JE. Impact of $16 \mathrm{~S}$ rRNA gene sequence analysis for identification of bacteria on clinical microbiology and infectious diseases. Clin. Microbiol. Rev. 17(4), 840-862 (2004).

42. Chakravorty $\mathrm{S}$, Helb D, Burday M et al. A detailed analysis of $16 \mathrm{~S}$ ribosomal RNA gene segments for the diagnosis of pathogenic bacteria. J. Microbiol. Methods 69(2), 330-339 (2007).

43. Li H, Zhang Y, Li DS et al. Comparisons of different hypervariable regions of rrs genes for fingerprinting of microbial communities in paddy soils. Soil Biol. Biochem. 41(5), 954-968 (2009).

44. Misbah S, Hassan H, Yusof MY et al. Genomic species identification of Acinetobacter of clinical isolates by $16 \mathrm{~S}$ rDNA sequencing. Singapore Med. J. 46(9), 461 (2005).

45. La Scola B, Gundi VA, Khamis A et al. Sequencing of the rpoB gene and flanking spacers for molecular identification of Acinetobacter species. J. Clin. Microbiol. 44(3), 827-832 (2006).

-• This study was the first work to identify Acinetobacter isolates at the species level using rpoB sequence analysis.

46. Lee MJ, Jang SJ, Li XM et al. Comparison of rpoB gene sequencing, 16S rRNA gene sequencing, gyrB multiplex PCR, and the VITEK2 system for identification of Acinetobacter clinical isolates. Diagn. Microbiol. Infect. Dis. 78(1), 29-34 (2014).

47. Khosravi AD, Sadeghi P, Shahraki AH et al. Molecular methods for identification of acinetobacter species by partial sequencing of the rpoB and 16S rRNA genes. J. Clin. Diagn. Res. 9(7), DC09 (2015).

48. Vaneechoutte M, De Baere T, Nemec A et al. Reclassification of Acinetobacter grimontii Carr et al. 2003 as a later synonym of Acinetobacter junii Bouvet and Grimont 1986. Int. J. Syst. Evol. Microbiol. 58(4), 937-940 (2008).

49. Wang J, Ruan Z, Feng Y et al. Species distribution of clinical Acinetobacter isolates revealed by different identification techniques. PLoS ONE 9(8), e104882 (2014).

50. Gundi VA, Dijkshoorn L, Burignat $S$ et al. Validation of partial rpoB gene sequence analysis for the identification of clinically important and emerging Acinetobacter species. Microbiology 155(7), 2333-2341 (2009).

51. Ahmed SS, Alp E. Genotyping methods for monitoring the epidemic evolution of A. baumannii strains. J. Infect. Dev. Ctries. 9(04), 347-354 (2015).

52. Nowak A, Burkiewicz A, Kur J. PCR differentiation of seventeen genospecies of Acinetobacter. FEMS Microbiol. Lett. 126(2), 181-187 (1995).

53. Nowak A, Kur J. Differentiation of seventeen genospecies of Acinetobacter by multiplex polymerase chain reaction and restriction fragment length polymorphism analysis. Mol. Cell Probes 10(6), 405-411 (1996).

54. Chen TL, Siu LK, Wu RC et al. Comparison of one-tube multiplex PCR, automated ribotyping and intergenic spacer (ITS) sequencing for rapid identification of Acinetobacter baumannii. Clin. Microbiol. Infect. 13(8), 801-806 (2007).

55. Soo PC, Tseng CC, Ling SR et al. Rapid and sensitive detection of Acinetobacter baumannii using loop-mediated isothermal amplification. J. Microbiol. Methods 92(2), 197-200 (2013).

56. Turton JF, Woodford N, Glover J et al. Identification of Acinetobacter baumannii by detection of the blaOXA-51-like carbapenemase gene intrinsic to this species. J. Clin. Microbiol. 44(8), 2974-2976 (2006).

-. This study was the first to describe the role of class $\mathrm{D} b \mathrm{blaxA}_{\mathrm{O}-51}$-like as an intrinsic gene which can be used as a biomarker for identification of $A$. baumannii at the species level.

57. Evans BA, Amyes SG. OXA $\beta$-lactamases. Clin. Microbiol. Rev. 27(2), 241-263 (2014).

58. Chen TL, Lee YT, Kuo SC et al. Emergence and distribution of plasmids bearing the blaOXA-51-like gene with an upstream ISAba1 in carbapenem-resistant Acinetobacter baumannii isolates in Taiwan. Antimicrob. Agents Chemother. 54(11), 4575-4581 (2010).

59. Lee YT, Kuo SC, Chiang MC et al. Emergence of carbapenem-resistant non-baumannii species of Acinetobacter harboring a blaOXA-51-like gene that is intrinsic to A. baumannii. Antimicrob. Agents Chemother. 56(2), 1124-1127 (2012).

60. Ahmadi A, Salimizand H. Delayed identification of Acinetobacter baumannii during an outbreak owing to disrupted blaOXA-51-like by ISAba19. Int. J. Antimicrob. Agents 50(1), 119-122 (2017).

61. Zander E, Higgins PG, Fernández-González A et al. Detection of intrinsic blaOXA-51-like by multiplex PCR on its own is not reliable for the identification of Acinetobacter baumannii. Int. J. Med. Microbiol. 303(2), 88-89 (2013).

62. Higgins PG, Wisplinghoff $\mathrm{H}, \mathrm{Krut} \mathrm{O}$ et al. A PCR-based method to differentiate between Acinetobacter baumannii and Acinetobacter genomic species 13TU. Clin. Microbiol. Infect. 13(12), 1199-1201 (2007).

63. Higgins PG, Lehmann M, Wisplinghoff $\mathrm{H}$ et al. gyrB multiplex PCR to differentiate between Acinetobacter calcoaceticus and Acinetobacter genomic species 3. J. Clin. Microbiol. 48(12), 4592-4594 (2010).

64. Croxatto A, Prod'hom G, Greub G et al. Applications of MALDI-TOF mass spectrometry in clinical diagnostic microbiology. FEMS Microbiol. Rev. 36(2), 380-407 (2012).

65. Ge MC, Kuo AJ, Liu KL et al. Routine identification of microorganisms by matrix-assisted laser desorption ionization time-of-flight mass spectrometry: Success rate, economic analysis, and clinical outcome. J. Microbiol. Immunol. Infect. 50(5), 662-668 (2017). 
66. Singhal N, Kumar M, Kanaujia PK et al. MALDI-TOF mass spectrometry: an emerging technology for microbial identification and diagnosis. Front. Microbiol. 6, 791 (2015).

67. Angeletti S et al. Matrix assisted laser desorption time of flight mass spectrometry (MALDI-TOF MS) in clinical microbiology. J. Microbiol. Methods. 138, 20-29 (2017).

68. Marí-Almirall M, Cosgaya C, Higgins PG et al. MALDI-TOF/MS identification of species from the Acinetobacter baumannii (Ab) group revisited: inclusion of the novel A seifertii and A. dijkshoorniae species. Clin. Microbiol. Infect. 23(3), 210-e211 (2017).

69. Espinal P, Seifert H, Dijkshoorn L et al. Rapid and accurate identification of genomic species from the Acinetobacter baumannii (Ab) group by MALDI-TOF MS. Clin. Microbiol. Infect. 18(11), 1097-1103 (2012).

70. Šedo O, Nemec A, Kř́žzová L et al. Improvement of MALDI-TOF MS profiling for the differentiation of species within the Acinetobacter calcoaceticus-Acinetobacter baumannii complex. Syst. Appl. Microbiol. 36(8), 572-578 (2013).

71. Hsueh Lu-Cheng Kuo, Tsung-Chain Chang et al. Evaluation of the bruker biotyper matrix-assisted laser desorption ionization-time of flight mass spectrometry system for identification of blood isolates of Acinetobacter species. J. Clin. Microbiol. 52(8), 3095-3310 (2014).

72. Kishii $\mathrm{K}$, Kikuchi $\mathrm{K}$, Matsuda $\mathrm{N}$ et al. Evaluation of matrix-assisted laser desorption ionization-time of flight mass spectrometry for species identification of Acinetobacter strains isolated from blood cultures. Clin. Microbiol. Infect. 20(5), 424-430 (2014).

73. Sousa C, Botelho J, Silva L et al. MALDI-TOF MS and chemometric based identification of the Acinetobacter calcoaceticus-Acinetobacter baumannii complex species. Int. J. Med. Microbiol. 304 (5-6), 669-677 (2014).

74. Chang HC, Wei YF, Dijkshoorn L, et al. Species-level identification of isolates of the Acinetobacter calcoaceticus-Acinetobacter baumannii complex by sequence analysis of the 16S-23S rRNA gene spacer region. J. Clin. Microbiol. 43(4), 1632-1639 (2005).

75. Yang Q, Rui Y. Two Multiplex Real-Time PCR Assays to Detect and Differentiate Acinetobacter baumannii and Non-baumannii Acinetobacter spp. Carrying blaNDM, blaOXA-23-Like, blaOXA-40-Like, blaOXA-51-Like, and blaOXA-58-Like Genes. PLoS ONE. 11(7), e0158958 (2016).

76.

76. Álvarez-Buylla A, Culebras E, Picazo JJ. , , . Identification of Acinetobacter species: is Bruker biotyper MALDI-TOF mass spectrometry a good alternative to molecular techniques? Infect Genet Evol. 12((2), ), 345-349 ( (2012).).

77.

77. Lee SY, Shin JH, Kim SH , , et al.Evaluation of matrix-assisted laser desorption ionization-time of flight mass spectrometry-based VITEK MS system for the identification of Acinetobacter species from blood cultures: comparison with VITEK 2 and MicroScan systems. . Ann Lab Med. 35((1), ), 62-68 ( (2015).).

78.

78. Pailhoriès H, Daure S, Eveillard M , et al.Using Vitek MALDI-TOF mass spectrometry to identify species belonging to the Acinetobacter calcoaceticus - Acinetobacter baumannii complex: a relevant alternative to molecular biology? Diagn Microbiol Infect Dis. 83((2), ), 99-104 ( (2015).).

79 .

79. Jeong S, Hong JS, Kim JO , et al.Identification of Acinetobacter species using matrix-assisted laser desorption ionization-time of flight mass spectrometry. . Ann Lab Med. 36((4), ), 325-334 ( (2016).). 
